\title{
Kidney function in the very low birthweight infant
}

In recent years there have been considerable advances in understanding the mechanisms of how the kidney grows and develops, which have shed light on the aetiology of some renal malformations and physiology. ${ }^{1}$ During fetal life the excretory role of the kidney is minimal and even the anephric fetus enjoys biochemical homoeostasis. Fetal urine production begins at about 10 weeks of gestation increasing progressively to $28 \mathrm{ml} /$ hour at term. Birth immediately imposes an excretory and fluid conservatory role on the neonatal kidney. The term baby is well adapted for the demands of postnatal life. In contrast the very low birthweight (VLBW) baby's renal function is more immature and the maintenance of normal fluid and electrolyte balance is difficult. Significant hyponatraemia (serum sodium concentration less than $130 \mathrm{mmol} / \mathrm{l}$ ) occurs in up to $43 \%$ of babies less than 30 weeks' gestational age. ${ }^{2}$ The difficulties of maintaining electrolyte homoeostasis are further compounded because the volume of distribution of sodium changes with extracellular fluid volume decreasing from $62 \%$ at 16 weeks to $43 \%$ at term. ${ }^{3}$ The VLBW infant is, therefore, in a state of relative overhydration. In the sick VLBW infant with asphyxia, sepsis, intracranial haemorrhage, pulmonary disease or renal failure, high circulating concentrations of antidiuretic hormone and atrial natriuretic factor make electrolyte requirements more unpredictable. A better understanding of the renal function of the VLBW infant is needed if appropriate management strategies are to be developed and the series of papers by Barry Wilkins in this edition of the journal sheds further light on some aspects of their renal adaptation. $4-7$

Nephrogenesis proceeds under a complex series of controls and is complete at 36 weeks' gestation with the full compliment of 1 million nephrons. After birth glomerular filtration rate increases in a preordained way from a mean of $0.6 \mathrm{ml} / \mathrm{minute}$ at 26 to $1.4 \mathrm{ml} /$ minute at 33 weeks' postconceptional age. Uncomplicated respiratory distress syndrome does not affect this maturation. Plasma creatinine concentration, which is generally used as a simple measure of glomerular filtration rate, is of limited value in the VLBW infant because of the wide normal ranges and the presence in the plasma of substances which interfere with its measurement. In these studies Dr Wilkins has measured true plasma creatinine but even so the normal ranges are wide, making the diagnosis of renal insufficiency difficult.

Hyponatraemia is a common problem in VLBW infants. ${ }^{8}$ Theoretically it may result from excessive sodium loss, water retention, or redistribution of sodium within body fluid spaces. Its management is controversial: some suggesting water restriction, others sodium supplementation. Correct management requires knowledge of the plasma and urine urea, creatinine, electrolytes, their volumes of distribution in body fluid spaces, and urine flow rates and these studies provide information about them. Timed urine collections are difficult to obtain and the formula derived by Wilkins: urine flow rate, $\mathrm{V}=90 \cdot 5 / \mathrm{urine}$ creatinine $(\mu \mathrm{mol} / \mathrm{l})$ could prove useful to tailor urine, water and electrolyte losses, although information based upon single urine samples must be interpreted with caution because of the large fluctuation in urine flow rates observed in these babies. Using this formula a wide range of urinary sodium losses up to $21 \mathrm{mmol} / \mathrm{kg} /$ day was observed, lending support to those who recommend sodium supplementation. However, VLBW infants are in a state of relative hypervolaemia and the increased urinary losses may be physiological. ${ }^{3}$ Measurement of the extracellular fluid and circulatory blood volumes would help to resolve this dilemma, but there is no simple reliable technique to do so. Body weight changes are imprecise in ventilated babies with intravenous catheters and infusion lines. High plasma urea concentrations are not helpful either as they may reflect increased catabolism rather than dehydration.

There is still much we do not understand about the renal function of the VLBW baby. A better understanding of the physiological changes is needed to improve the management of the fluid and electrolyte problems they present. This series of papers by Wilkins provides some helpful observations that will be useful to tailor the fluid and electrolyte management of individual babies to their requirements.

Department of Paediatrics and Child Health,

J T BROCKLEBANK

St fames's University Hospital,

Leeds LS9 $7 T F$

1 Bard JBL, Woolf AS. Nephrogenesis and the development of renal disease. Nephrol Dial Transplant 1992;7:563-72.

2 Al-Dahhan J, Haycock GB, Chantler C, Stimmler L. Sodium homeostasis in term and preterm neonates/renal aspects. Arch Dis Child 1983;58:335-42.

3 Friis-Hanson B. Body water compartments children: changes during growth and related changes in body composition. Pediatrics 1961;28:169-81.

4 Wilkins BH. Renal function in sick very low birthweight infants: 1 . glomerular filtration rate. Arch Dis Child 1992;67:1140-5.

5 Wilkins BH. Renal function in sick very low birthweight infants: 2. urea and creatinine excretion. Arch Dis Child 1992;67:1146-53.

6 Wilkins BH. Renal function in sick very low birthweight infants: 3. sodium, potassium, and water excretion. Arch Dis Child 1992;67:1154-61.

7 Wilkins BH. Renal function in sick very low birthweight infants. 4. Glucose excretion. Arch Dis Child 1992;67:1 162-5.

8 Haycock GB, Aperia A. Salt and the newborn kidney. Pediatr Nephrol 1991;5:65-70. 